\title{
X-RAY STUDY OF CLUSTERS OF GALAXIES WITH ASCA
}

\author{
T. OHASHI \\ Department of Physics, Tokyo Metropolitan University \\ 1-1 Minami-Ohsawa, Hachioji, Tokyo 192-03, Japan
}

\section{Introduction}

$\mathrm{X}$-ray emission from clusters of galaxies is the most useful tool in studying mass distribution and chemical compositions in these enormously large systems. The hot intracluster medium (ICM) has been heated up to $k T=3-10$ $\mathrm{keV}$ during the gravitational collapse, and X-ray luminosities indicate that the gas is more massive than the total galaxy mass contained in clusters by factors of 3-5. This makes ICM the dominant form of baryons in the universe. In many clusters observations indicate that ICM is in a hydrostatic equilibrium within a potential governed by the dark matter, and the cooling time is longer than the Hubble time except for the bright centers. The ICM, therefore, enables us a close look at the structure of gravitational potential. At the same time, heavy-element abundances in the ICM and their distribution are used to estimate past supernova activities and metal injection mechanism in cluster space.

The Japanese X-ray astonomy observatory ASCA (Tanaka et al. 1994) was launched in February 1993 and is still actively observing almost all categories of objects. ASCA has a high spectral resolution and a wide energy range from 0.5 to $10 \mathrm{keV}$. Because of its moderate angular resolution with a half power diameter of about $3^{\prime}$, extended objects such as clusters of galaxies are very suitable targets for ASCA. Spatially resolved energy spectra of clusters, obtained for the first time, almost directly show us hot gas structure and heavy element distribution. In this brief review, I shall describe recent ASCA results on bright near-by clusters for which internal structures are well-resolved. The results presented here are obtained with the GIS instrument (Ohashi et al. 1996, Makishima et al. 1996). I do not go into results from distant clusters, for whidh readers should refer to other reviews. 

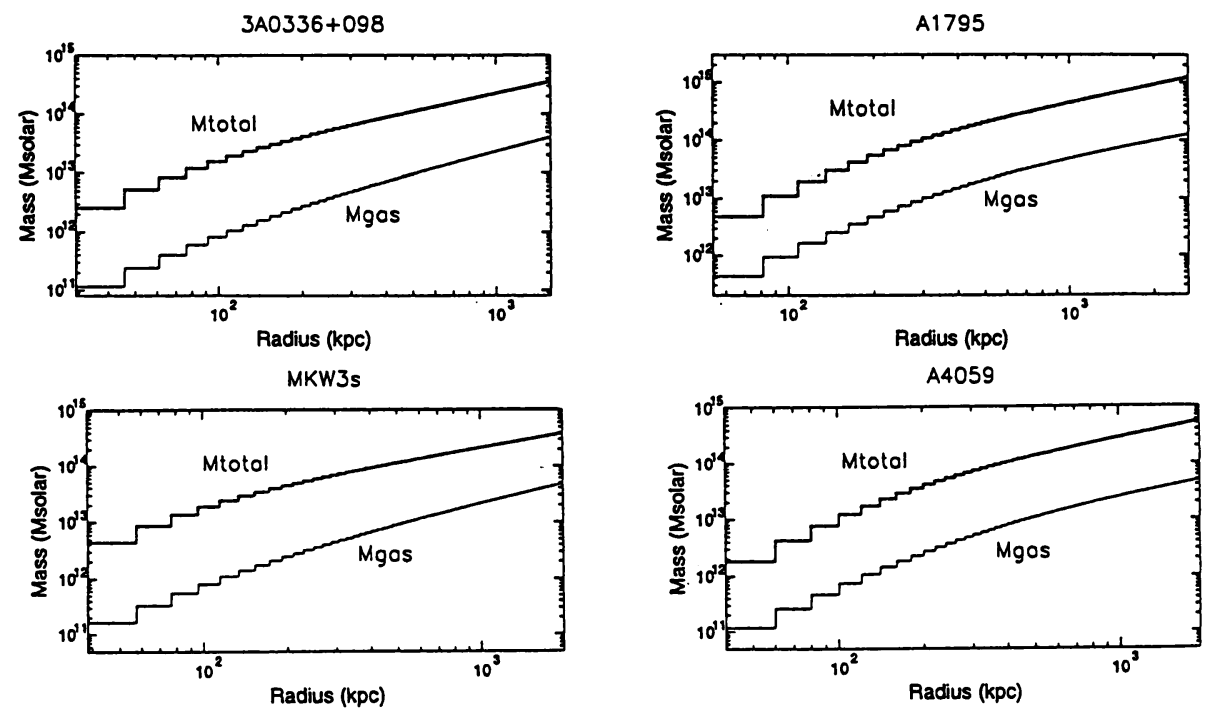

Figure 1. ASCA results of gas mass and total mass plotted as a function of radius for 4 clusters. These results are obtained by K. Kikuchi.

\section{Mass Distribution}

Radial distribution of X-ray surface brightness in clusters, except for sharp central peaks around $\mathrm{cD}$ galaxies, is well described by so-called $\beta$-models. In this model radial distribution of electron density $n_{e}$ in a 3 -dimensional space is described by,

$$
n_{e}=n_{0}\left\{1+\left(\frac{r}{r_{c}}\right)^{2}\right\}^{-\frac{3}{2} \beta}
$$

where $n_{0}$ is the central value, $r_{c}$ is the core radius $(100-300 \mathrm{kpc})$, and $\beta$ denotes the parameter taking $0.5-0.7$ in clusters. We can assume that the hot gas is in a hydrostatic equilibrium, i.e. the gas is virialized in the potential well whose structure is dominated by the dark matter. This is supported from smooth distribution of X-ray brightness and a fairly uniform gas temperatures. Then the gravitational mass can be estimated as,

$$
M_{\mathrm{total}}(<r)=-\frac{k T r}{\mu m_{\mathrm{H}} G}\left(\frac{\partial \ln n}{\partial \ln r}+\frac{\partial \ln T}{\partial \ln r}\right),
$$

where $k$ and $G$ are the Boltzmann and gravitational constants, $m_{H}$ is the hydrogen mass, $\mu$ is the mean molecular weight $(\approx 0.6)$, respectively.

Examples of the integrated mass profile are shown in Fig. 1.

The measured ratios of the gravitational mass, $M_{\text {total }}$, to the gass mass, $M_{\text {gas }}$, turn out to be similar from cluster to cluster, and the fraction of 


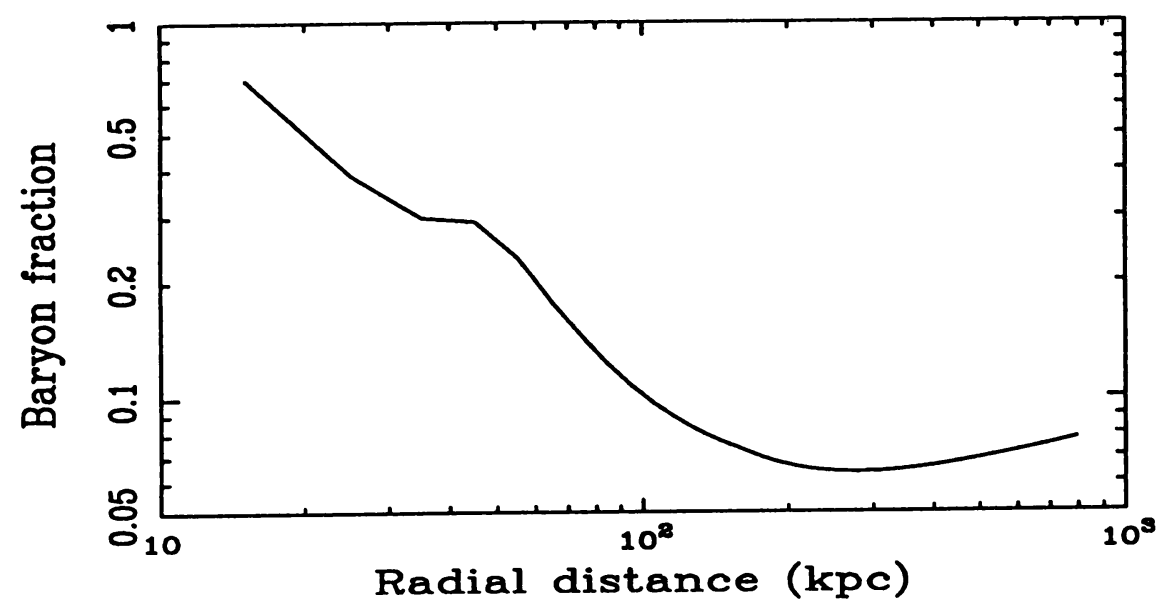

Figure 2. Radial variation of baryonic fraction in the Centaurus cluster derived by lkebe (1996).

baryon which is the sum of $M_{\text {stellar }}$ (stellar mass which is roughly $20 \%$ of $M_{\text {gas }}$ in rich clusters) and $M_{\text {gas }}$ divided by $M_{\text {total }}$ becomes around 0.2 . ASCA and ROSAT observations indicate that the baryonic fraction is between $15 \%$ and $30 \%$ for rich and poor clusters, and there seems no systematic variation of baryonic fraction with the cluster richness (Böhringer 1994, David et al. 1994). Since clusters have been formed by collecting matter within several times $10 \mathrm{Mpc}$, it is argued that no significant segregation of baryon from dark matter is possible in such a large scale (White et al. 1993). Therefore, it is likely that the observed baryon fraction is the cosmic average, i.e. the value of $\Omega_{B}$ would be around 0.2 .

The primordial nucleosynthesis theory and recent measurements of light element abundances indicate $0.009 h^{-2} \leq \Omega_{B} \leq 0.02 h^{-2}$ (Copi et al. 1995). If one combines this with the cluster results, $\Omega \sim 0.3$ is obtained. There are, in fact, other supporting evidences for the low $\Omega$ universe (see e.g. Bahcall 1995, Dekel 1994). I must say, however, that clusters occupy a tiny amount of volume (probably a few \%) in the universe, and we do need to observe the gravitational mass and baryon mass in supercluster scales since there is clearly a structure (non uniformity in mass distribution) in this scale. We hope that future sensitive X-ray observations, such as Xray absorption measurements for distant quasars (see Aldcroft et al. 1994) or study of peculiar motions of clusters (Bahcall 1997), would be able to constrain both baryonic and total masses in supercluster scales.

Looking closely at the cluster center, we notice that the baryonic fraction, $f_{B}=\left(M_{\text {stellar }}+M_{\text {gas }}\right) / M_{\text {total }}$, is not constant with radius. As shown 
in Fig. 2 (from Ikebe 1996), $f_{B}$ is greater than 0.3 in the center due to the large contribution from stellar mass. It then drops to less than 0.1 at a radius of a few $100 \mathrm{kpc}$ and rises to $0.2-0.3$ in the very outer region because of the flatter distribution of hot gas than dark matter (see David et al. 1995). This scale-dependent segregation of baryon is obviously common to all clusters and probably related to fundamental properties of dark matter.

\section{Cluster Center}

The central peak in the X-ray emission in clusters has been always interpreted in terms of cooling flow models (see Fabian 1994). Since the cooling time of the gas $\left(t_{\text {cool }} \propto n_{e}^{-1}\right)$ is shorter than the Hubble time, the gas should cool down by radiation without the presence of additional heat input. However, ASCA observations are revealing new features besides the existence of cool gas with $k T \sim 1 \mathrm{keV}$ in the central region.

The first finding is the apparent co-existence of the hot and cool components in the center. The peak of the X-ray emission is composed of both hot and cool components. This feature is first indicated in the Centaurus cluster by Fukazawa et al. (1994) and subsequently confirmed by Ikebe (1996) and Ikebe et al. (1998). In the 3 dimensional model assuming a pressure balance between the hot and cool components, ASCA data require that the filling factor of the cool part is about $50 \%$ in the center and drops to 0 at $r=50 \mathrm{kpc}$. An important feature is that strong gradient in the metal abundance is clearly seen from $\mathrm{Fe}-\mathrm{K}$ lines in the hot component. It seems likely that the metal-rich gas has its origin in the $\mathrm{cD}$ galaxy, then the hot component filling the cluster center is comprizing mostly the gas injected from the $\mathrm{cD}$ galaxy. It may be that part of the metal rich gas, which was originally cool at around $1 \mathrm{keV}$ at the time of injection, has been heated up due to thermal conduction.

The central region of Hydra-A cluster has been examined by Ikebe et al. (1997). In particular, simulataneous fitting of ROSAT and ASCA data indicates that two-temperature plasma is necessary within $50 \mathrm{kpc}$ of the cluster center as shown in Fig. 3. The cool component is a minor part, and the X-ray peak is mostly caused by the hot component. Since the mass of the cool component is a few times $10^{10} M_{\odot}$, it could be the interstellar matter contained in the $\mathrm{cD}$ galaxy. Another cause of the cool component is due to cooling flows. The mass deposition rate in the Hydra-A cluster has been previously estimated as $600 M_{\odot} \mathrm{yr}^{-1}$ from the Einstein IPC data by attributing all the central peak to cooling flows (David et al. 199?). By applying this model only to the true amount of the cool component derived here, the mass deposition rate drops to as low as $60 M_{\odot} \mathrm{yr}^{-1}$. Therefore, detection of the hot and cool components in the cluster center requires us 
(a)

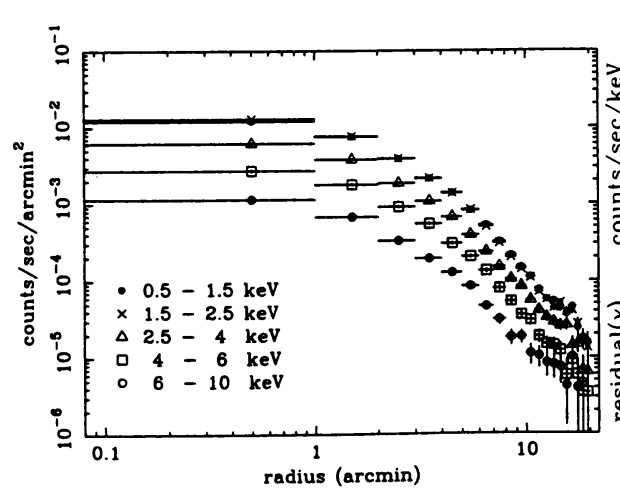

(b)

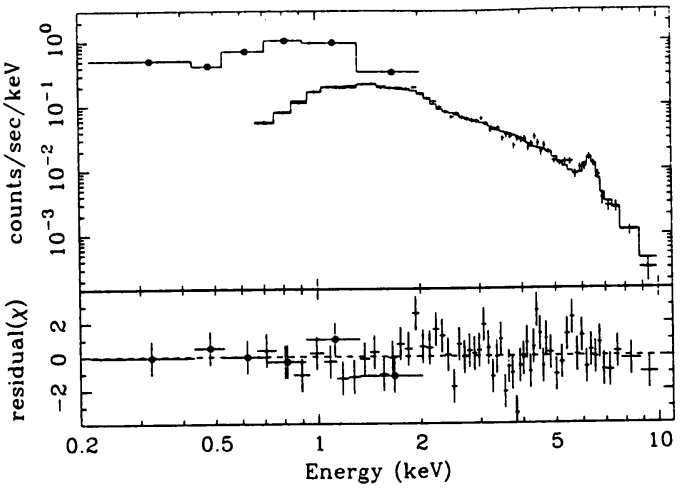

Figure 3. (a) Radial profiles of Hydra-A in 5 different energy bands with GIS, showing similar characteristics. (b) Simultaneous fit of the PSPC (filled circles) and GIS (crosses) pulse-height spectra for the central $1.5^{\prime}$ region, requiring 2 temperatures. The results are taken from Ikebe et al. (1997)

to reconsider the scenario of cooling flow models. Xu et al. (1998) report similar results on $\mathrm{A} 1795$.

The hot component in the center of clusters (Centaurus, Hydra-A, A1795) is most likely to be gravitationally bound in the system, since it has the same temperature as the surrounding region. The centrally peaked profile in the hot component implies that there is a potential dimple caused by a dark matter concentration in the center. Such a cusp structure in the dark matter distribution is also suggested from the gravitational lenzing effect in several clusters: In those cases, the lens mass estimated from observed features of arcs and arclets in cluster cores turns out to be larger than that determined from X-ray observations by a factor of 2-3. The lens mass is obtained within about $100 \mathrm{kpc}$ from the cluster center where arcs and arclets are present. In larger scales, the mass determined from weak lens effect (distortion of the images of background galaxies) suggests better agreement with the X-ray mass within $30 \%$.

The reason why strong lens always gives much higher mass than the $\mathrm{X}$ ray one is still unclear. As for the X-ray result, the $\beta$ model approximation is probably incorrect if one goes inside of the core radius as shown very recently by Tamura (1998) for A1060. He shows, based on a detailed analysis of the ROSAT PSPC and the ASCA data, that gravitational mass needs to be more centrally peaked than the standard $\beta$ profile. The gravitational potential probably has a cusp structure such as that suggested from recent numerical works by Navarro, Frenk and White (1997). On the other hand, 
the mass determined from strong lenses can be overestimated if there is a non-spherical distribution in the gravitating matter, even though this effect cannot easily explain the factor of 2 discrepancy. We hope that a future extensive analysis of the ASCA data, supplying good temperature and metallicity information, combinted with a high resolution image from ROSAT would reveal a detailed mass structure in the central region.

\section{Metal Abundance}

ASCA's unique power is its capability of measuring the metallicity distribution in clusters. This information constrains the scenario of galaxy evolution and the process of metal deposition. Figure 4 shows the radial distribution of measured metal abundance for 6 clusters. Some clusters, such as Centaurus and Virgo, show strong enhancement near $\mathrm{cD}$ galaxies. Since the plotted data are not corrected for the point-spread function of the X-ray telescope of ASCA, actual abundance profile should be significantly narrower and concentrated within 20-30 kpc. These features indicate that $\mathrm{cD}$ galaxy produces large amount of metal in some clusters. We should also note that the timescale for the iron sedimentation is inversely proportional to the density and estimated to be longer than the Hubble time.

The metallicity distribution in larger scales has not been measured with enough sensitivity. The surface brightness drops rapidly in the outer regions, and the difficulty in ASCA is that correction for the stray light effect is very large. In AWM7, mapping observations from ASCA show interesting feature in the metal distribution. Fig. 5 shows the radial distribution of metal abundance after corrections for stray light. To interpret the observed feature, we assume that iron (which mostly controls the observed abundance) mass density follows the $\beta$ model whose core radius is same as that of gas. The gas distribution indicates its $\beta=0.58$, and we expect iron to have a larger $\beta$ value because of the abundance gradient. As shown in Fig. 5, $\beta$ values around 0.8 for iron desribes the abundance profile well. Galaxy distribution in clusters has been investigated and recent results show that it is described by $\beta$ models with $\beta=0.8-0.9$. Therefore, our AWM7 result implies that iron mass distribution is well tracing galaxy distribution. This naturally indicates that the main source of metals in the ICM are galaxies, and also that metals are not strongly mixed within the cluster (see Ezawa et al. 1997 and Ezawa 1998 for details).

Another recent ASCA result is a finding of a systematic correlation between Si abundance and ICM temperature (Fukazawa et al. 1998). As shown in Fig. 6, abundances of $\mathrm{Fe}$ and $\mathrm{Si}$ show very different behavior with cluster richness (i.e. ICM temperatures). The Fe abundance shows a rather tight concentration around 0.3 solar (here, 1 solar abundance of $\mathrm{Fe}$ 
$\mathrm{A} 1060$

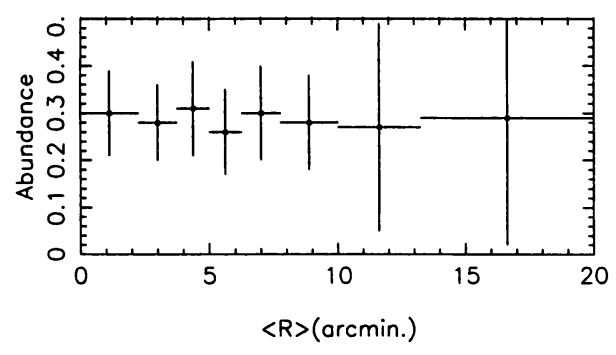

Virgo

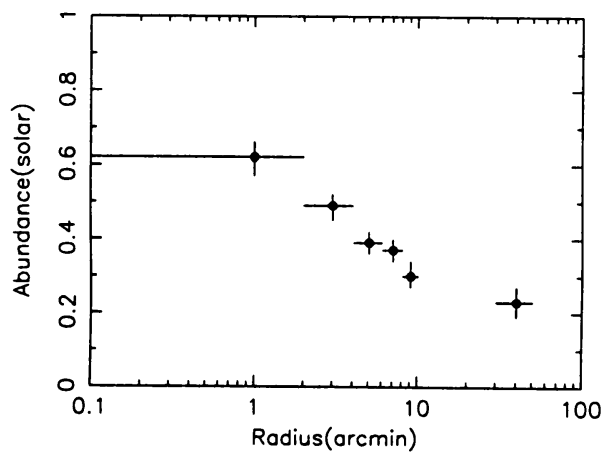

$\mathrm{A} 1795$

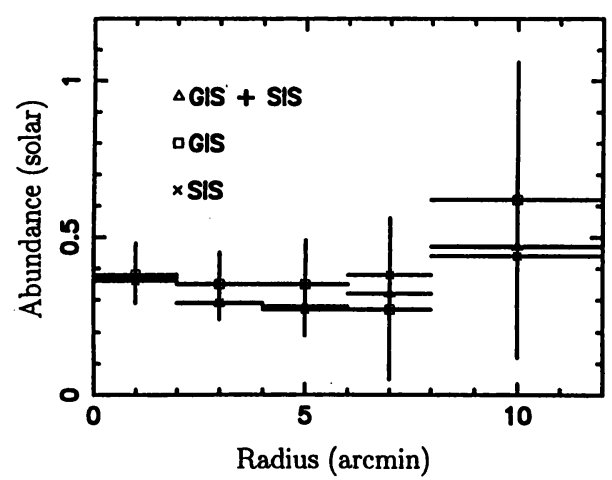

Hydra-A

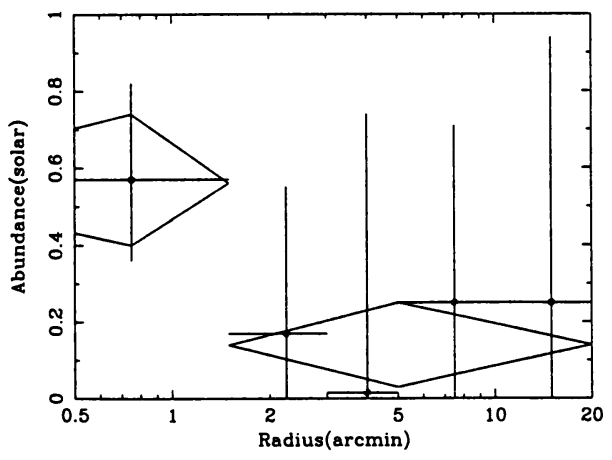

Centaurus

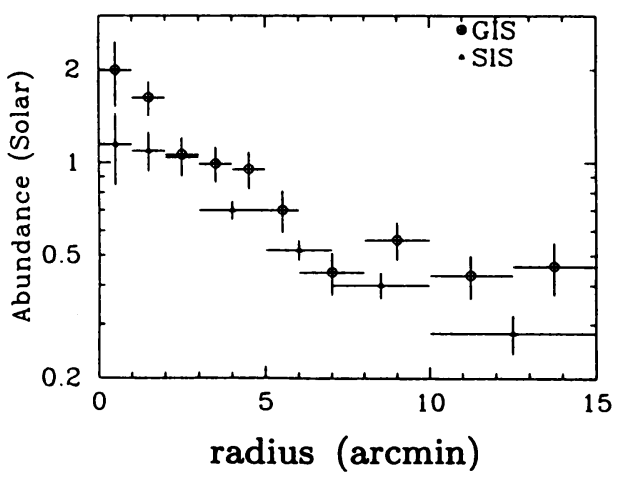

$3 \mathrm{~A} 0336+098$

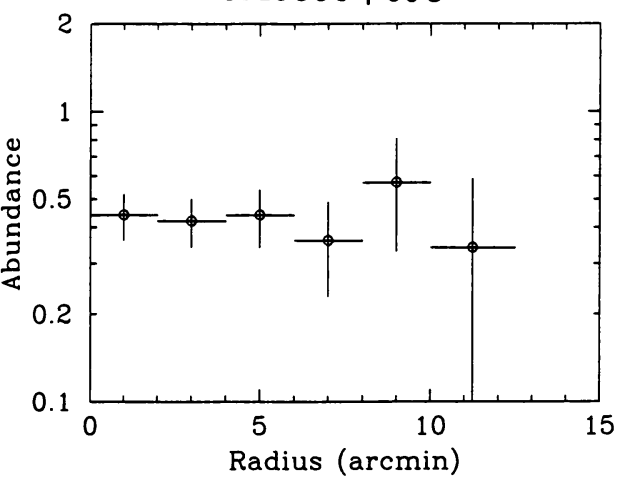

Figure 4. Collection of the radial abundance profiles for 6 clusters measured with ASCA. References are A1060: Tamura et al. (1996), Hydra-A: Ikebe et al. (1997), Virgo: Matsumoto et al. (1996), Centaurus: Fukazawa et al. (1994), A1795: Xu et al. (1998), 3A0335+096: private communication from Y. Fukazawa and K. Kikuchi. 


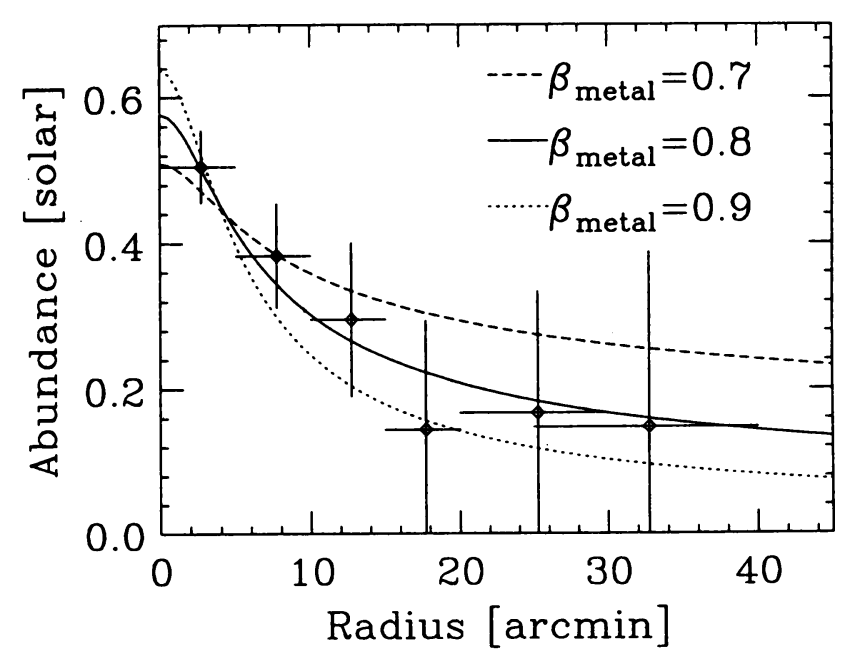

Figure 5. A large scale abundance gradient recently discovoered in AWM7 by Ezawa et al. (1997)

(a)

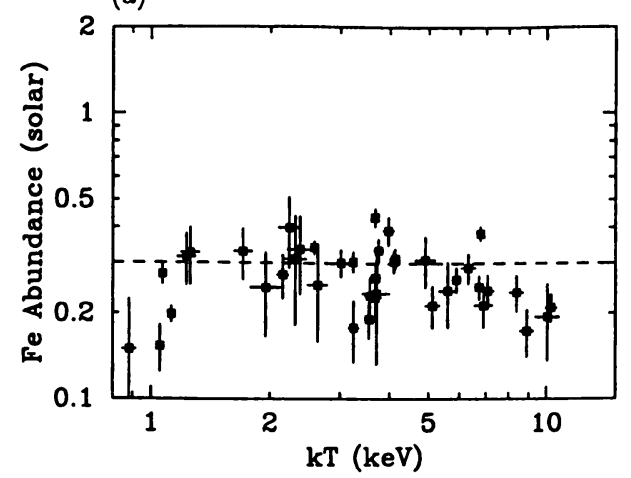

(b)

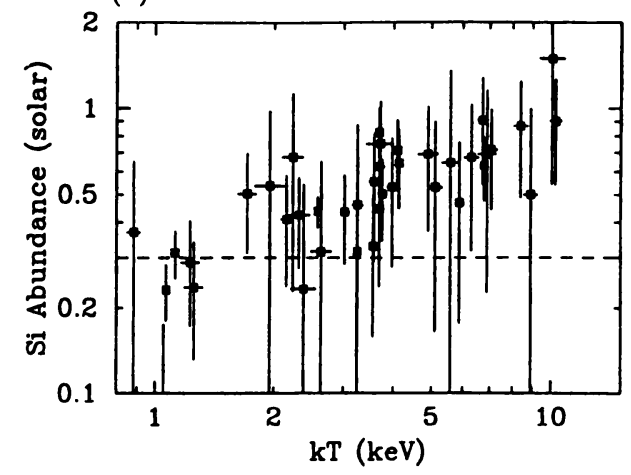

Figure 6. Average metal abundance in clusters plotted against the ICM temperature for (a) Fe and (b) Si obtained by Fukazawa et al. (1998).

is $4.7 \times 10^{-5}$ in number) for ICM temperatures between 1.5 and $7 \mathrm{keV}$. In contrast to this, $\mathrm{Si}$ shows significantly higher abundance for $k T>4$ $\mathrm{keV}$ by a factor of $\sim 2$. The $\mathrm{Si}$ abundance drops to less than 0.5 solar at $k T<3 \mathrm{keV}$. Since equivalent width (EW) of Si emission lines rapidly drops with temperature, we have to show that the abundance change is real against systematic errors. From observations of various X-ray sources, spurious features in the Si-line energy are less than $4 \mathrm{eV}$ in EW. This is much less than the observed $\mathrm{EW}$ of $20 \mathrm{eV}$, and systematic error in $\mathrm{Si}$ 
abundance is less than $30 \%$ for $k T<6 \mathrm{keV}$.

Since $\mathrm{Si}$ is expected to be produced in type-II supernovae in galaxy formation phase and injected into a cluster space by galactic winds. Much of $\mathrm{Fe}$, on the other hand, is later supplied from type-Ia supernovae by stripping or slow dissipation. The low $\mathrm{Si}$ abundance in poor clusters suggest that high-velocity wind gas would have escaped from a shallow potential. In rich clusters, all the metal-rich gas has been retained by a deep potential. Therefore, the observed abundance feature supports the view that a large amount of metals has been injected in an early epoch with galactic winds.

The author acknowledges support from Yasushi Fukazawa, Hajime Ezawa, Ken'ichi Kikuchi, and Yasushi Ikebe for providing results prior to publication.

\section{References}

Aldcroft, T., Elvis, M., McDowell, J., \& Fiore, F. (1994) Astrophys. J., 437, 584

Bahcall, N.A. (1995) Ann. N.Y. Acad. of Sci., 759, 636

Bahcall, N.A. (1997) Unsolved Problems in Astrophysics, ed. J.N. Bahcall and J.P. Ostriker, Princeton Univ. Press, p. 61

Böhringer, H. (1994) Cosmological Aspects of Clusters of Galaxies

Copi, J.C., Schramm, D.N., and Turner, M.S. (1995) Science, 267, 192

David, L.P., Jones, C., and Forman, W. (1995), ApJ, 445, 578

David, L.P., Arnaud, K.A., Forman, W., \$ Jones, C. (1990), Astrophys. J., 356, 32

Dekel, A. 1994, Ann. Rev. Astron. Astrophys., 32, 371

Ezawa, H. (1998) Ph. D. thesis, University of Tokyo

Ezawa, H., Fukazawa, Y., Makishima, K., Ohashi, T., Xu, H., \& Yamasaki, N.Y. (1997) ApJ Letters, 490, L33

Fabian, A.C. (1994) Ann. Rev. Astron. Astrophys., 32, 277

Fukazawa, Y. et al. (1994) Publ. Astron. Soc. Japan, 46, L55

Fukazawa, Y. et al. (1998) Publ. Astron. Soc. Japan, 50, 187

Ikebe, Y. (1996) Ph. D. Thesis, University of Tokyo (RIKEN IPCR CR-87)

Ikebe, Y. et al. (1997) Astrophys. J., 481, 660

Ikebe, Y. et al. (1998) Astrophys. J., submitted (ISAS RN-613)

Matsumoto, H. et al. (1996) Publ. Astron. Soc. Japan, 48, 201

Navarro, J.F., Frenk, C.S., \& White, S.D.M. (1997), Astropnys. J., 490, 493

Ohashi, T. et al. (1996) Publ. Astron. Soc. Japan, 48,

Tamura, T. (1998) Ph. D. Thesis, University of Tokyo

Tamura, T. et al. (1996) Publ. Astron. Soc. Japan, 48, 671

Tanaka, Y., Inoue, H., and Holt, S.S. (1994), Publ. Astron. Soc. Japan

White, S.D.M., Navarro, J.F., Evrard, A.E., \& Frenk, C.S. (1993) Nature, 366, 429

Xu, H. et al. (1998), Astrophys. J., in press 